\title{
New insights into the clinical evaluation of hereditary transthyretin amyloidosis patients: a single center's experience
}

REVIEW

This article was published in the following Dove Press journal:

Degenerative Neurological and Neuromuscular Disease

28 August 2012

Number of times this article has been viewed

Ole B Suhr'

Sandra Gustavsson'

Victoria Heldestad ${ }^{2}$

Rolf Hörnsten ${ }^{3}$

Per Lindqvist ${ }^{1}$

Erik Nordh ${ }^{2}$

Urban Wiklund ${ }^{4}$

'Department of Public Health and Clinical Medicine, ${ }^{2}$ Department of Pharmacology and Clinical Neuroscience, ${ }^{3}$ Department of Surgical and Perioperative Sciences, Clinical Physiology, Heart Centre,

${ }^{4}$ Department of Radiation Sciences, Biomedical Engineering, Umeå University, Umeå, Sweden
Correspondence: Ole B Suhr Department of Medicine, Umeå University Hospital, SE 90I 85 Umeå, Sweden

$\mathrm{Tel}+4690785 \quad 1383$

Fax +4690143986

Email ole.suhr@medicin.umu.se
Abstract: Over the last decade, new medical treatment modalities have emerged based on increased insights into amyloid formation. With the increased possibilities for treatment of amyloidosis caused by transthyretin (TTR) amyloid deposits comes the need for diagnostic procedures for early diagnosis and better tools to follow disease progression. This is of particular importance in clinical trials evaluating the efficacy of new treatments. Until recently, the treatment of TTR amyloidosis (ATTR) was based solely on liver transplantation, a procedure that has halted disease progression in many patients. Liver transplantation has been especially effective in patients under the age of 50 years carrying the TTR V30M mutation, whereas the outcome of the procedure has been variable for others, particularly elderly male patients and those carrying a non-V30M mutation. This review concentrates on new insights derived from our center's experience with liver transplantation, how to implement this experience in evaluation of new treatment modalities for ATTR, and how to facilitate early diagnosis of neuropathy with easily available diagnostic tools. Attention has focused on manifestations of the disease that involve the heart and the peripheral nervous system; change in peripheral nerve function has been the primary endpoint in two controlled clinical trials, one finished and one ongoing. New insights into the amyloid formation process and the lessons learned from liver transplantation give the opportunity to design potentially effective treatment modalities for ATTR. It appears reasonable to suspect that a combination of different treatment modalities may be required to treat the disease, and that different treatment regimes will be designed according to the phenotype of the disease. For the patients and their relatives there is now a solid foundation for optimism, with prospects of several effective medical treatment possibilities within the coming decade.

Keywords: neuropathy, cardiomyopathy, amyloid, diagnostic tools, new treatment modalities

\section{Introduction}

Transthyretin (TTR) amyloid contains misfolded TTR that has assembled into beta-pleated sheets: amyloid fibrils. It is generally believed that mutations in the TTR gene alter the stability of the TTR tetramer, leading to formation of misfolded monomers that assemble into amyloid fibrils. ${ }^{1,2}$ More than 100 amyloidogenic mutations have been identified, ${ }^{3,4}$ with the most common probably being the V122I, which has a prevalence of close to $4 \%$ in the African-American population. ${ }^{5}$ The V122I mutation gives rise to cardiomyopathy caused by amyloid deposition in the myocardium, whereas another common mutation, V30M, is primarily associated with a peripheral neuropathy. However, it should be noted that wild-type TTR can assemble into amyloid, giving rise to cardiomyopathy that generally occurs after the seventh decade of life and with a male predominance. ${ }^{6}$ Interestingly, in the vervet monkey the V122I 
mutation has been found in aged animals that developed cardiomyopathy and heart failure; thus, the V122I mutation is naturally occurring in humans and monkeys, and it causes cardiomyopathy in both species. ${ }^{7}$

The clinical presentation of TTR amyloidosis (ATTR) is extremely varied, not only between mutations but also between different geographic populations carrying the same mutation. ${ }^{8-10}$ In addition, variation in phenotype within a geographic area has been described for the V30M mutation. ${ }^{11,12}$ The disease is relentlessly progressive and the survival rate is generally between 10 and 15 years. ${ }^{13-16}$

Since liver transplantation was introduced as a treatment for the disease, knowledge has expanded further. It has become clear that no improvement has been noted for certain mutations after liver transplantation, especially in patients with symptoms from the central nervous system (CNS) and those whose foremost complications involve the heart, whereas for other mutations, particularly the V30M mutation in younger patients, the progress of the disease has been halted. ${ }^{17-26}$ In patients with TTR amyloid cardiomyopathy, combined liver and heart transplantations have been performed with encouraging results. ${ }^{27,28}$ Considering the risk that the patient takes by undergoing a liver transplantation, the failure to halt disease progression in many cases, the shortage of organs available for transplantation, and the need for transplant patients to be on lifelong immune suppression therapy, the need for effective medical treatments is conspicuous.

Evaluation of the efficacy of a treatment must rely on outcome measurements. To achieve reliable outcome measurements in patients with ATTR has proven to be difficult: the disease has a very variable phenotype, and improved survival - the ultimate measurement of efficacy - takes considerable time to validate in a disease with an expected survival rate of 10 years or more. To overcome this lack of validated outcome measures in ATTR, recent double-blind studies of a TTR-stabilizing agent, tafamidis meglumine, have employed rating scales developed for scoring of neuropathy in diabetes mellitus as markers of disease progression. ${ }^{29,30}$ However, a neurological scale is probably not a useful tool for patients with dominant cardiomyopathy.

This review will concentrate on new insights into amyloid fibril composition and its impact on the disease phenotype, derived from our center's experience with ATTR amyloidosis. In addition, medical treatment and tools for early recognition of neurological impairment will be discussed.

The authors performed a search for published papers within the area of ATTR and its complications, including cardiomyopathy, autonomic and peripheral neuropathy, and liver transplantation: the search tools used were PubMed and Web of Science.

\section{New insights into amyloid fibril composition}

Histopathological examination of amyloid deposits has revealed the existence of two different forms of TTR deposits: one with a strong congophilic reaction and the other with a weaker affinity for Congo red..$^{31,32}$ The weak staining has been noted for patients with wild-type ATTR but has also been noted for some V30M patients. Further analysis by Western blot showed that the differences in affinity for Congo red was associated with differences in fibril composition: full-length TTR amyloid fibrils were associated with a strong affinity for Congo red, whereas weak affinity was noted in deposits containing a mixture of full-length and truncated TTR amyloid fibrils. ${ }^{31,33}$ Examination of the fibril composition from different organs showed that the composition was similar in all tissues from the same individual. Therefore, fibril composition in material obtained from subcutaneous fat was similar to that found in the heart. ${ }^{33}$ Importantly, the difference could be linked to the phenotype of the disease, where the mixed fibril type was found in late-onset V30M patients as well as in senile systemic amyloidosis patients, and the full-length TTR fibrils were found in early-onset V30M patients. In addition, the mixed fibril type was associated with cardiomyopathy, whereas full-length fibrils were found in patients where neuropathy dominated the clinical picture. ${ }^{33}$

Since cardiomyopathy has been a problem after liver transplantation in late-onset V30M patients, especially males, who have a phenotype resembling that of senile systemic amyloidosis, the possibility that amyloid fibril composition may have an effect on posttransplant cardiomyopathy has been explored. Analysis of amyloid deposits in transplant patients showed that wild-type TTR was rapidly incorporated in TTR amyloid deposits in patients with mixed fibrils, whereas this process was significantly slower in patients with full-length fibrils. ${ }^{34}$ Deterioration in heart function after transplantation was observed for patients with mixed fibrils, whereas no such deterioration was noted for patients with full-length fibrils. ${ }^{21}$ In addition, mixed fibrils were found in T60A amyloidosis patients and liver transplantation has not been successful for this mutation, with a rapid development of cardiomyopathy after the procedure. ${ }^{35}$ Thus, amyloid fibril composition appears to be related to outcome following liver transplantation for ATTR. The individual's type of amyloid fibril composition remains the same over time and is not changed by transplantation. ${ }^{34}$ 
The differences in fibril composition raise some questions: is the amyloid formation process in ATTR the same for the two different forms of fibrils? Are differences in outcome of treatment modalities related to fibril composition? These questions should be taken into consideration in future evaluations of treatment modalities.

\section{Clinical expressions of ATTR Variations in phenotype}

ATTR is often divided into two phenotypes, characterized by the main targeted organ: (1) the heart leading to a hypertrophic/restrictive cardiomyopathy or (2) the peripheral nervous system leading to neuropathy. However, mutations with oculoleptomeningeal amyloidosis with symptoms from the CNS have also been described. ${ }^{36-39}$ The vast variation in ATTR is intriguing, and currently there is no explanation why different organs are targeted in different mutations.

V30M, one of the best-studied mutations, has displayed an unexpected variation in presenting symptoms and complications as well as variation in age of disease onset. ${ }^{8}, 10,11,40$ For Swedish patients, the median age at onset is 56 years, ${ }^{41}$ whereas for Portuguese, Brazilian, and Japanese patients, the onset in the endemic areas has been reported to be in the third decade of life. ${ }^{13,14}$ It was originally suspected that all V30M patients share a common Portuguese founder, but haplotype analysis has clearly shown that all Swedish patients share a common Swedish founder. ${ }^{42}$ Reports from Japan, Portugal, and France have described cases outside the endemic areas with a phenotype characterized by late onset (over 50 years of age) and often suffering from cardiomyopathy in addition to neuropathy. ${ }^{10,43}$ Even differences in nerve fiber losses have been noted between early- and late-onset cases: a predominance of small-fiber loss has been noted in early-onset cases while late-onset cases have been characterized by relative preservation of unmyelinated fibers. ${ }^{44} \mathrm{~A}$ study of Portuguese early- and late-onset cases has suggested that differences in genes coupled to proteins are associated with amyloid deposits, and an analysis of mitochondrial haplogroups has shown differences between early- and late-onset Swedish patients. ${ }^{43,45}$ Differences in maternally inherited mitochondrial DNA were also shown to be a possible explanation for the maternal anticipation noted for Portuguese families with the V30M mutation, but it could not solely explain the maternal anticipation observed in Swedish families with the V30M mutation. ${ }^{46}$ So far, no genetic factor has been identified that can with certainty be linked to the variation in disease phenotype for $\mathrm{V} 30 \mathrm{M}$ patients, or to the variation of phenotypes observed for the different TTR mutations.
Medical treatment for ATTR has been concentrated on V30M patients, with encouraging outcomes found in the tafamidis trial. ${ }^{29}$ Considering the low prevalence of non-V30M patients, it will probably be difficult to conduct studies targeting other ATTR variants; however, the differences in phenotype even for V30M amyloidosis should be taken into consideration when treatment studies are designed.

\section{Neuropathy}

\section{Somatic neuropathy: early screening}

Amyloidosis is often associated with peripheral neuropathy, and evaluations of peripheral nerve function bring important contributions to a comprehensive diagnosis of the disease. The most important means for objective assessment of the state of the peripheral nervous system are electroneurography $(\mathrm{ENeG})$ and needle electromyography (EMG), which evaluate the functional state of the large-diameter myelinated nerve fibers. However, the neuropathy found in V30M patients is initially predominantly characterized by affection of small-diameter nerve fibers, with clinical observations of disturbed temperature and pain perception, or signs of organ dysfunctions secondary to impaired autonomic innervation (see below). As electrophysiological testing primarily addresses the function of large myelinated fibers, patients may thus report marked symptoms of sensory impairment without showing any detectable abnormalities in ENeG or EMG assessment, occasionally even for periods of several years. This may critically delay the diagnosis and may lead to pronounced nerve damage before the patient receives adequate therapy.

The evaluation of peripheral small-diameter myelinated or unmyelinated fibers is more cumbersome, as there are no methods for direct recording from these fibers readily available for clinical routine use. Quantitative evaluation has to be achieved indirectly by studies of the effects of thindiameter fiber on the target organs $\mathrm{s}^{47,48}$ that require advanced equipment and which, because of this requirement, are not suitable for widespread screening of patients with minor symptoms. Likewise, the elaborate routines needed may not be mastered at nonspecialized centers and would be difficult to utilize for follow-up purposes at most centers. Skin biopsy for nerve density quantification has been utilized in ATTR patients with advanced neuropathy, and has been found to correlate with disease severity. ${ }^{49}$ However, the method has not been tested for early diagnosis of ATTR neuropathy and it requires neuropathological expertise not readily available at most centers. In addition, decreased blood flow in peripheral 
tissues of ATTR patients with ensuing impaired wound healing makes the method less attractive. ${ }^{50}$

Another well-established rationale for early identification of small-diameter fiber polyneuropathy is thermal quantitative sensory testing (QST), which is based on the patient's thermal detection capacity - as determined by psychophysical assessments of the thermal perception thresholds (TPTs). ${ }^{51-59}$ Such testing is routinely performed at specialist clinics, but a more general use of thermal QST in patients with possible amyloidosis has been hampered by the fact that a TPT is dependent on a series of compound underlying somatosensory mechanisms, ranging from the peripheral sensors over the ascending pathways to the central processes contributing to thermal perception. ${ }^{60,61}$ Several alternatives have been suggested for thermal QST, essentially divided into reactiontime-inclusive and reaction-time-exclusive methods. ${ }^{52,62}$ For practical reasons, the reaction-time-inclusive "method of limits" (MLI) can be recommended for clinical use, as it comprises a quick, reliable, and easily adopted rationale well understood by both examiners and patients. ${ }^{62-69}$

When tested with the MLI, a group of Swedish ATTR patients with genetically and biopsy-verified disease, having clinical symptoms of polyneuropathy but lacking electrophysiological signs of nerve fiber abnormalities, showed significantly increased perception thresholds for cold and warmth compared with controls. The patients also showed more abnormal thresholds at distal test sites than the controls (see Figure 1). ${ }^{70}$ These findings are concordant with the notion of a small-diameter fiber involvement early in the course of the disease. However, it is important to note that the QST must be performed bilaterally in the legs and arms, and preferably with both distal and proximal test locations, at least in the legs. Such a testing strategy is crucial for a firm interpretation of the test results, as asymmetric alterations in peripheral sensory detection capacity may also be caused by other nerve affections, such as impingements of peripheral nerves or spinal roots, ${ }^{71,72}$ or by referred sensations originating from altered segmental sensory processing. ${ }^{73}$ Hence, to confirm a putative small-diameter fiber neuropathy, the QST should indicate bilateral threshold elevations with distal predominance, paralleling the clinically characteristic "stocking and glove" distribution of the sensory disturbances. Such alterations in TPTs of the extremities therefore strongly corroborate the presence of small-diameter peripheral neuropathy or a debuting generalized peripheral neuropathy.

In a general outpatient or nonspecialist center setting where screening of unselected patient materials is available, a more basic approach must be adopted. To this end, testing of temperature discrimination with two traditional metal cylinders, one cold and one warm, rolled over the skin bilaterally at distal and proximal test sites is suggested. The cylinders should have suggested suprathreshold stimulus temperatures of $25^{\circ} \mathrm{C}$ (cold) and $40^{\circ} \mathrm{C}$ (warm), and the patient should be blinded to the presentation and repetition order.

QST utilizing the method of MLI appears to be attractive for the early diagnosis of neuropathy in ATTR, since it detects abnormalities before ENeG and EMG. It should also be suited for follow-up purposes in clinical trials. So far, no improvement of neurological impairment has been noted in transplant patients, either by QST or by electrophysiological testing.

\section{Autonomic neuropathy}

Autonomic disturbances are commonly encountered in ATTR and may be the presenting manifestation of the disease. However, measurement of the autonomic nervous system is challenging, and few methods are available that offer the possibility for early detection of autonomic neuropathy or of grading the disturbances. The intricate direct intraneural micrographic recordings of autonomic nerve fiber activity, ${ }^{74}$ laboratory evaluation of adrenergic "spillover," 75 and detailed quantitative evaluations of sweating ${ }^{47,76,77}$ are not readily available for clinical use but have shown promising outcomes for diagnosis of amyloidosis-related autonomic neuropathy in a study by the Mayo Clinic. ${ }^{78}$ However, the widely used galvanic skin response (or somatosensory evoked response) test is less reliable, as it cannot be used for the grading of autonomic activity - it can only inform whether or not a galvanic skin response is present.

Analysis of heart rate variability (HRV) has proven to be a valuable method for detecting and measuring autonomic impairment in ATTR patients. ${ }^{79-82}$ Two different methodologies have been used for evaluating cardiac autonomic function before and after liver transplantation for ATTR: (1) as a short-term HRV recording (up to 30 minutes) in a controlled laboratory environment with the patient placed on a manually operated tilt table ${ }^{83}$ or (2) as a long-term HRV recording using 24-hour electrocardiographic monitoring (Holter monitoring). ${ }^{79,80}$ Studies of Swedish patients have not shown any improvement in cardiac autonomic function after liver transplantation, but studies have also shown that the development of cardiac arrhythmias continues after liver transplantation. ${ }^{79,80}$ The latter is a major confounder when HRV recordings are evaluated in ATTR patients, where it is presumed that the heart rate fluctuations originate from activity in the autonomous nervous system. The present 


\section{Stimulation}

sites

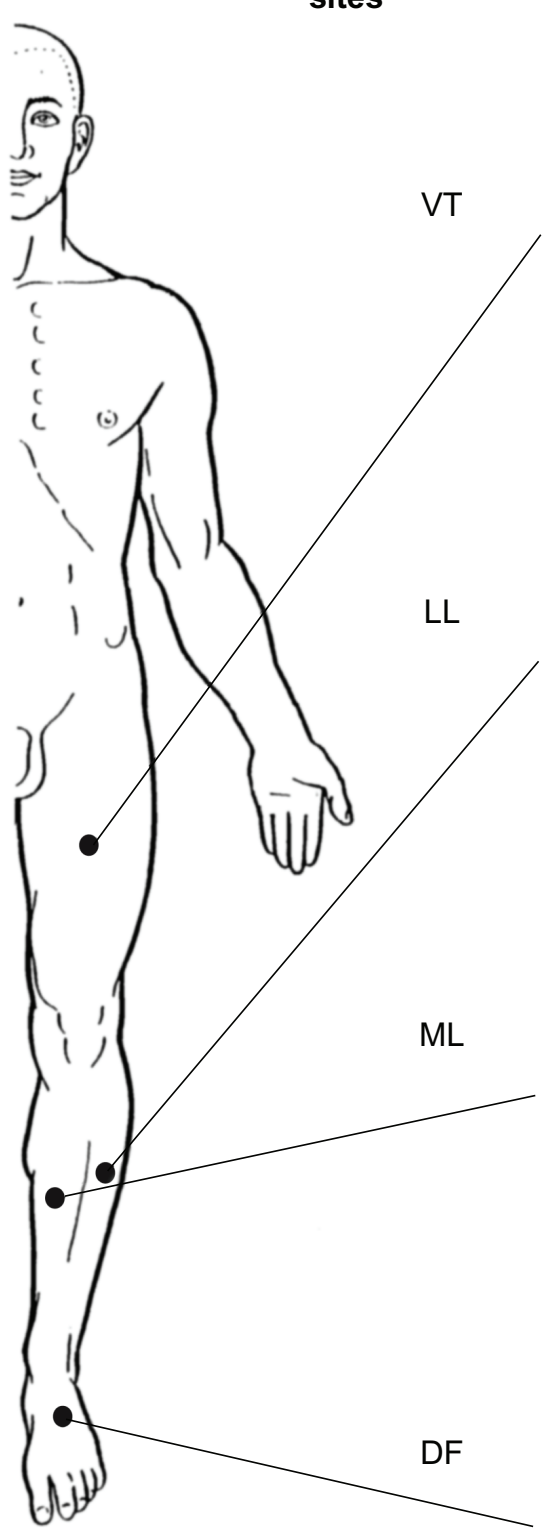

CT FAP

CT controls
WT FAP

WT Controls

\section{Thermal thresholds}
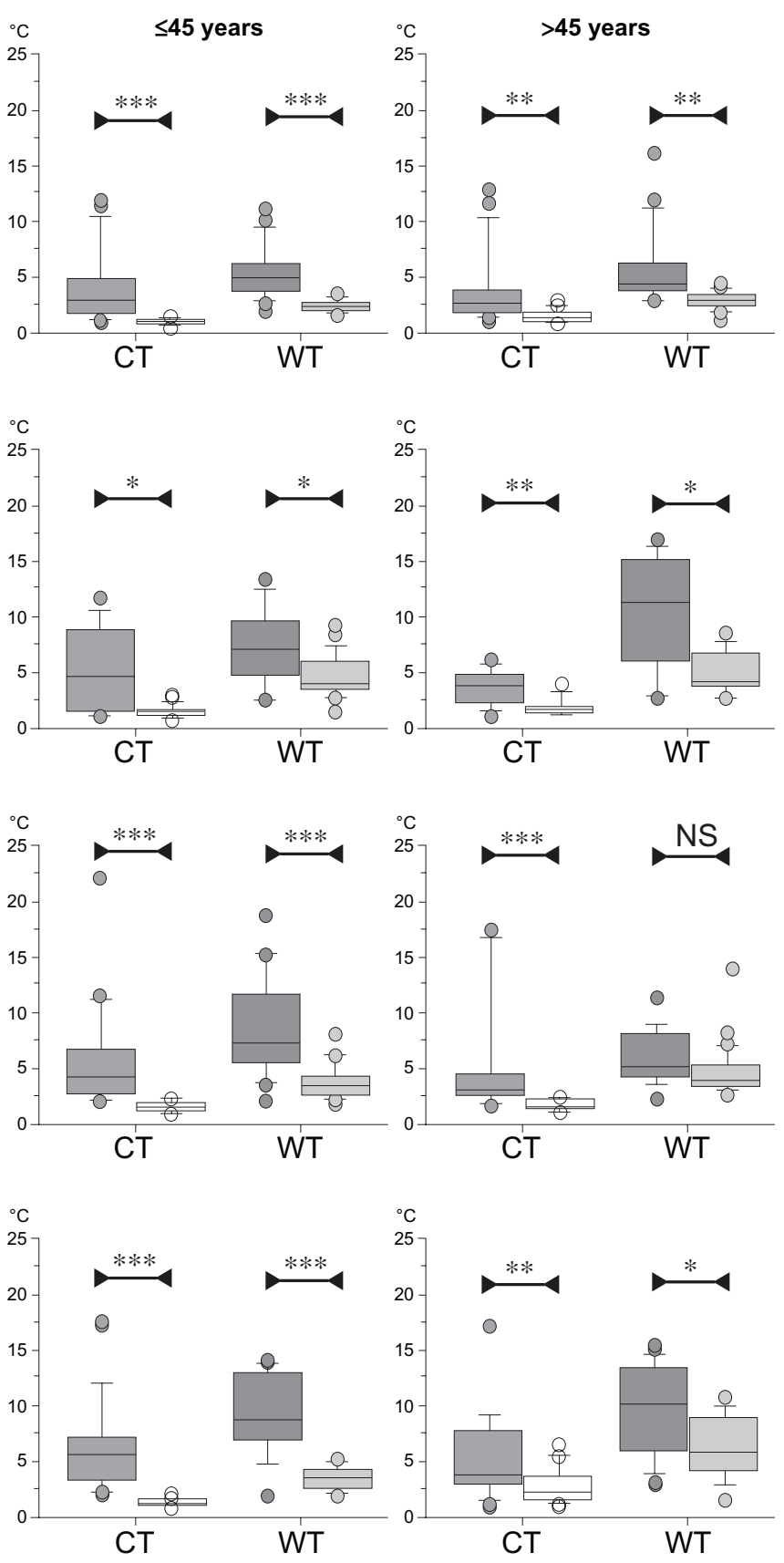

Figure I Box plots of thermal thresholds in a group of transthyretin V30M amyloidosis patients with no sign of electrophysiological abnormalities (familial amyloid polyneuropathy [FAP]; $n=23$ ) and a reference group (controls; $n=43$ ), at the dorsum of the foot (DF), medial $(M L)$ and lateral $(L L)$ parts of the lower leg, and ventral part of the thigh (VT). Thermal thresholds are expressed as change in degrees Celsius (absolute values) from an adapted starting temperature of $32^{\circ} \mathrm{C}$.

Notes: Boxes show median, 25th, and 75th percentiles, and circles show outliers below/above the 10/90 percentiles. Note the increased thermal thresholds in FAP patients, most prominent at distal test sites. *, ***,***.

Figure reproduced from Heldestad and Nordh, ${ }^{70}$ with permission from the publisher.

Abbreviations: $C T$, cold threshold; NS, not significant; WT, warm threshold. $* P<0.05$; $* * P<0.01$; ***P $<0.00$ I

authors have found that many patients present subtle atrial arrhythmias that lead to increased HRV, falsely indicating normal autonomic function. ${ }^{82,84,85}$ Therefore, all HRV recordings in ATTR patients must be carefully investigated to detect subtle atrial arrhythmias.
In the tafamidis study of V30M amyloidosis patients, the deep breathing test was selected as an integrated part of a scoring system for nerve function. The test is performed as a short sequence with controlled deep breathing at a rate of six breaths per minute, but since no previous study has 
presented any data from this test in ATTR patients, the reliability of the test is questionable. Figure 2 shows three examples of heart rate responses during the deep breathing test taken from the authors' database of recordings in Swedish patients. Patient A showed the expected response during deep breathing with the same fluctuations in heart rate as in the breathing signal (six cycles per minute), although there were only small respiratory-related fluctuations in heart rate during spontaneous breathing. Patient B had even more reduced HRV during spontaneous breathing but presented supraventricular escape beats during deep breathing, resulting in large fluctuations in heart rate that at first glance appeared to be related to the breathing pattern. Finally, patient $\mathrm{C}$ also lacked HRV during spontaneous breathing but presented an irregular atrial arrhythmia during deep breathing. The authors have observed this pattern in many ATTR patients - the deep breathing test triggers arrhythmias. Therefore, interpretation of HRV recordings and the subsequent analysis require good knowledge of the methods and its limitations, particularly in patients presenting high indices of HRV.

Recently, a marked overshoot in blood pressure was observed after tilt reversal in ATTR patients. ${ }^{86}$ The study was based on 15 ATTR patients and 14 healthy subjects, showing a significant inverse relationship between blood pressure overshoot and HRV, as well as an overshoot in four of the five patients who had arrhythmia. Thus, this investigation could provide an alternative method for the assessment of autonomic neuropathy in patients with cardiac arrhythmia ${ }^{86}$ Finally, in addition to autonomic dysregulation, HRV abnormalities can also be found in patients with cardiac amyloidosis. ${ }^{87}$

\section{The heart}

Heart complications are important in ATTR diseases. They consist primarily of a restrictive-hypertrophic cardiomyopathy caused by amyloid deposits in the myocardium and/ or conduction disturbances caused by amyloid infiltrations in the conductive system of the heart. In addition, atrial and even ventricular arrhythmias may develop.

\section{Cardiomyopathy}

It is not unusual that patients under evaluation for ATTR present with increased wall thickness on echocardiographic examination but do not show any symptoms consistent with

\section{Patient A}

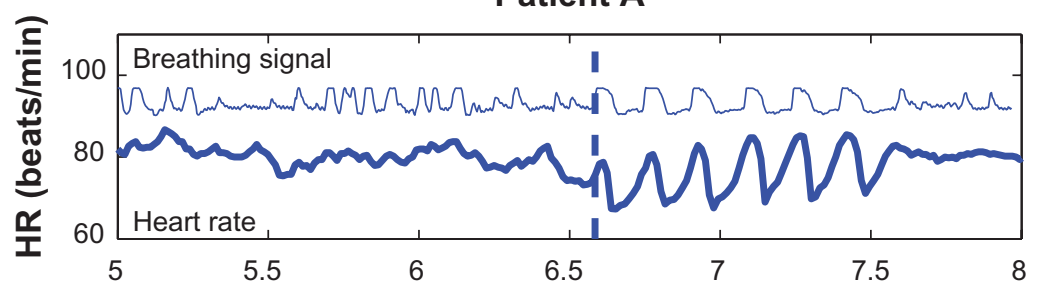

Patient B

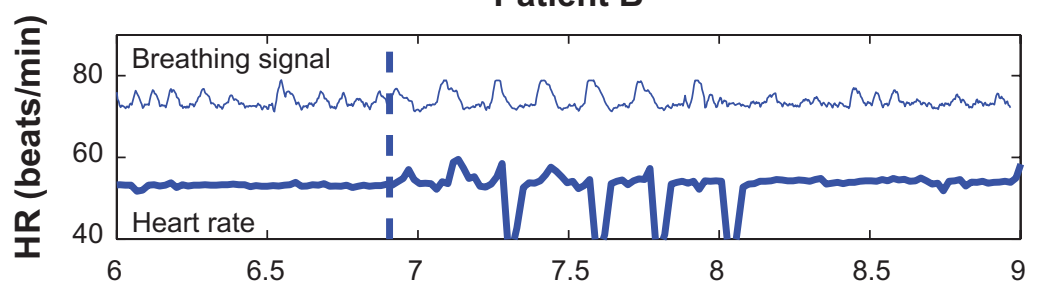

Patient C

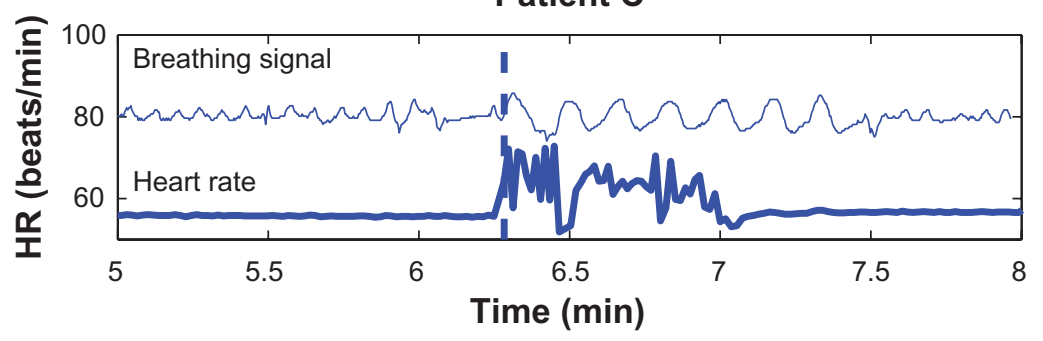

Figure 2 Recordings of heart rate (HR) variability patterns in three transthyretin amyloidosis patients during spontaneous and deep breathing, where the recordings were performed in the supine position. Patient A showed the normal response in HR during deep breathing, whereas patients $B$ and $C$ presented marked increases in HR variability because of cardiac arrhythmia during the test. 
heart failure ${ }^{88}$ Cardiomyopathy is more often encountered in elderly patients, particularly in males, or in patients with non-V30M mutations. ${ }^{12,89}$

It is difficult to use two-dimensional (2-D) echocardiography to differentiate amyloid disease from other causes of left ventricular hypertrophy, although it may detect signs of amyloid infiltration of the heart. Increased septal and posterior wall thickness, including in the right heart, with abnormal systolic and diastolic function are common findings in amyloid heart disease. Atrial involvement as well as thickened papillary muscles, valve leaflets, and small to moderate increased pericardial effusion should raise the suspicion of amyloid heart disease (Figure 3). ${ }^{90,91}$

The differentiation between cardiac amyloidosis and hypertrophic cardiomyopathy has clinical implications, since heart transplantation and new medical treatments are options for patients with TTR amyloid heart disease. St John Sutton et $\mathrm{al}^{92}$ found reduced radial systolic wall motion and reduced wall thickening during systole were highly specific for amyloid heart disease. Amyloid heart disease is also characterized by a high degree of highly reflective echoes that corresponds to nodules containing amyloid or a mixture of collagen and amyloid. ${ }^{90,91,93,94}$

Examination of the heart function by 2-D strain measurements disclosed impaired heart function in V30M patients, even before increased wall thickness or any restrictive filling pattern were present, ${ }^{95}$ but $2-\mathrm{D}$ strain measurements was

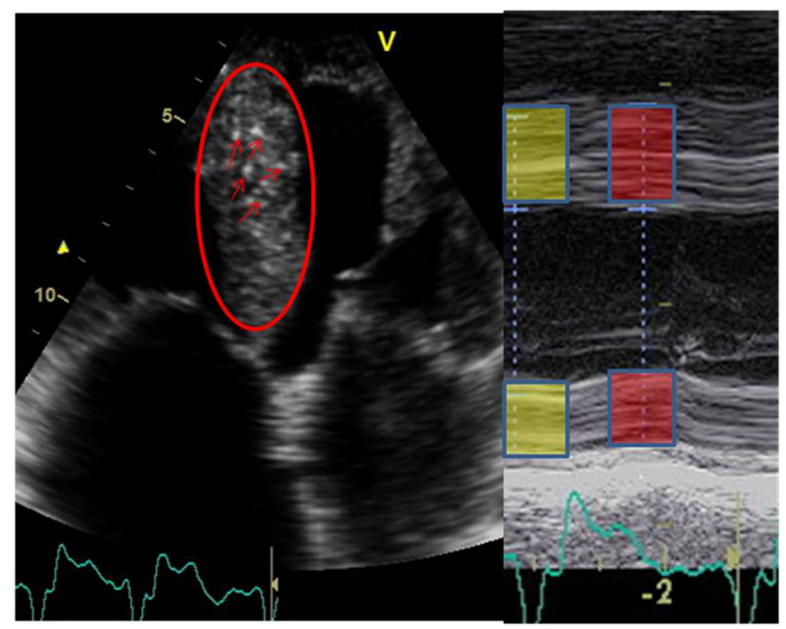

Figure 3 Typical patterns found in echocardiographic examinations in transthyretin amyloidosis patients.

Notes: Left panel: two-dimensional echocardiogram showing increased sparkling echoes or highly refractile echoes (detected at points indicated by red arrows) within the ventricular septum (indicated by red oval). Right panel: M-mode examination showing low septal and posterior wall thickening between diastole (yellow) and systole (red). In this case the wall is not thickening during systole but is being dragged into the left ventricular cavity by tethering from other segments. unable to differentiate between hypertrophic or amyloid cardiomyopathy. ${ }^{96}$

Global strain measurement of the heart function, derived from speckle tracking, was used to compare the heart function of patients with full-length TTR amyloid fibrils and patients with mixed fibril type after liver transplantation, and deterioration in heart function was able to be identified in the latter group. ${ }^{21}$ In addition, since the method is largely based on a computerized imaging program, it is not influenced by intraindividual variations to the same extent as manual measurements of interventricular septal thickness, which makes it attractive for follow-up studies of treatment modalities.

Three-dimensional (3-D) echocardiography is a technique that provides assessment of volumes, dyssynchrony, and 3-D strain. Migrino et $\mathrm{al}^{97}$ used 3-D echocardiography to compare patients with light-chain (AL) amyloidosis and healthy controls, and this showed left ventricular dyssynchrony in the AL amyloidosis patients. This technique still has the limitation of being highly dependent on good image quality, but it is under continual development and could become an important diagnostic tool in the future.

Even though echocardiography with the addition of new techniques should raise the suspicion of amyloid cardiomyopathy and although 2-D speckle tracking measurements appear to be suited for follow-up examinations, additional noninvasive tools are needed.

One very promising technique is scintigraphic examination, either by 99 mTc-3,3-diphosphono-1,2-propanodicarboxylic acid (DPD) ${ }^{98}$ or by 99 mTc-pyrophosphate. ${ }^{99,100}$ This technique has proven to have high sensitivity and specificity in the detection of TTR amyloid disease and is an important tool for differentiating between ATTR and other types of cardiomyopathy. However, scintigraphic examinations have not yet been developed for follow-up purposes.

Magnetic resonance investigation of the heart with gadolinium enhancement has been reported to disclose amyloid deposition within the myocardium and to give accurate estimation of heart dimensions. However, it does not appear to be as sensitive as DPD scintigraphy ${ }^{101}$ for the diagnosis of TTR amyloid deposits, and since it is more costly and cannot be used in patients with pacemakers, its use for diagnosis and follow-up purposes is limited.

Serum N-terminal pro-brain natriuretic peptide has proven to be a reliable indicator of heart involvement in AL amyloidosis, and to be useful for follow-up evaluation of disease treatment. ${ }^{102,103}$ The analysis could also be well suited for diagnosis and follow-up purposes in ATTR, since 
it correlates with echocardiographic measurements of heart function. Troponin examination appears to be less useful in TTR amyloid cardiomyopathy than in AL amyloidosis, ${ }^{103}$ but high-sensitivity troponin may prove to be more useful. ${ }^{104}$

For diagnosis, the suspicion of amyloid heart disease should arise from echocardiographic examination, and a DPD scintigraphic examination should be able to differentiate between TTR amyloid cardiomyopathy and other forms of cardiomyopathy. Echocardiography with 2-D strain measurements in combination with serum markers such as pro-brain natriuretic peptide are useful for follow-up examinations. Since heart complications are common in ATTR, removal of amyloid deposits for treatment of cardiomyopathy or substantially decreasing TTR levels in the plasma to diminish amyloid formation are attractive options. The benefit of tafamidis or other TTR stabilizers for heart function has not been proven in controlled trials; an open-label study is completed but the outcome is not yet published.

\section{Rhythm disturbances}

Amyloid deposits in the heart are the main cause of electrocardiographic disturbances. Findings include heavy amyloid infiltrations in the sinoatrial node, the atrial musculature, and the atrioventricular conduction system. ${ }^{105,106}$ Moreover, the heart rhythm can also be affected by the autonomic dysfunction commonly found in ATTR patients. ${ }^{107,108}$

Different cross-sectional studies, mainly based on conventional electrocardiograms (ECGs), have revealed a high prevalence of conduction disturbances, intermittent atrial and ventricular arrhythmias, and signs of low voltages in the ECGs. ${ }^{109-112}$ Development of severe arrhythmia is common in V30M patients. A longitudinal study of 12-lead ECG changes in non-transplant ATTR patients over a mean follow-up period of 8 years showed that $19(31 \%)$ of 61 patients required pacemaker implantation during the observation period. ${ }^{109}$ A study of 24-hour Holter ECG recordings showed a similar development of severe arrhythmia in transplant patients: pacemaker and intracardiac defibrillator implantation was performed in approximately $25 \%$ of patients over a median follow-up period of 11 years. ${ }^{113}$ Therefore, patients who have undergone liver transplantation should be regularly reexamined by Holter monitoring for arrhythmia necessitating pacemaker implantation. It should be noted that the recommendation for pacemaker implantation in ATTR patients deviates from recommendations for other patient groups, since the presence of an autonomic neuropathy diminishes the patient's ability to react to bradycardia. Even severe conduction disturbances may be asymptomatic, since episodes of bradycardia and/ or ventricular tachyarrhythmia are often noted at night when the patient is asleep, increasing the risk of nocturnal sudden death.

The development of conduction disturbances is not coupled with the development of cardiomyopathy, ${ }^{12}$ probably because the amyloid deposition in the conduction system does not follow deposition in the myocardium. ${ }^{101}$ The aforementioned subtle arrhythmias may be induced by amyloid deposition in the atrium and be related to cardiomyopathy; nonetheless, supraventricular arrhythmia is predominantly found in elderly patients. ${ }^{12,114}$

\section{Other organs commonly affected in ATTR}

Since ATTR is a systemic disease and amyloid deposits can be found in all organs, a variety of expressions of the disease are described for ATTR, and the outcome for different organ systems after liver transplantation varies, as may the response of these organ systems to medical treatment.

\section{Gastrointestinal}

Impaired gastrointestinal function is a common complication in ATTR. The mechanism is not fully understood, but autonomic and enteric nervous system impairments are probably involved. ${ }^{107,115,116}$ Unintentional weight loss, often before the patient complains of gastrointestinal disturbances, is frequently reported. ${ }^{14}$ The decline in the patient's nutritional status, measured by the modified body mass index (mBMI) (body mass index multiplied by serum albumin in grams per liter to compensate for edema) has been utilized. ${ }^{15,117}$ The mBMI correlates with survival and is also used as a predictor of mortality and morbidity after liver transplantation. It also serves as a secondary endpoint in the tafamidis and diflunisal clinical trials. ${ }^{29,118}$

No improvement in gastrointestinal function has been noted after liver transplantation, ${ }^{119}$ but a significantly improved mBMI was noted for patients treated with tafamidis compared with those receiving placebo. ${ }^{29}$ Thus, this index appears to be well suited for following patients in clinical studies and for evaluating the impact of treatment modalities.

\section{Kidney}

Both kidney and urinary bladder dysfunctions are common complications in ATTR. Impaired bladder function was coupled with an increased mortality after liver transplantation in a French study. ${ }^{17}$ Combined liver and kidney transplantation may be considered in patients with kidney failure. ${ }^{120,121}$ 
The kidney function appears to remain stable after liver transplantation. ${ }^{122}$

An increase in urinary tract infections was noted for patients treated with tafamidis compared with those receiving placebo. ${ }^{29}$

\section{Eyes}

A variety of eye complications are noted in ATTR, of which vitreous opacities and glaucoma are the most important. ${ }^{123}$ Neither of these two complications subsides after transplantation, since the retina synthesizes TTR. ${ }^{124,125}$ The development of glaucoma is troublesome, since the damage to the patient's vision is irreversible, and the impairment of vision can be substantial before the patient notes it.

The impact of tafamidis on eye complications has not yet been examined. However, the efficacy of the treatment depends on the compound's ability to travel into the eye and exert an effect in the intraocular environment. Again, this has not yet been examined for tafamidis, and neither antisense nor silencing RNA should penetrate from the circulation into the eye and affect TTR production.

\section{Carpal tunnel syndrome}

A peculiar feature of ATTR is the high incidence of carpal tunnel syndrome - this may occur before other symptoms of amyloid disease. ${ }^{3,126}$ It is often bilateral but, considering the high incidence of carpal tunnel syndrome in the general population, an active search for systemic amyloidosis, in the absence of other symptoms, is not indicated. However, for a patient with peripheral neuropathy or cardiomyopathy of unknown etiology, a history of carpal tunnel syndrome should raise the suspicion of ATTR. In addition, carpal tunnel syndrome is often found in patients with the rare oculoleptomeningeal form of ATTR. ${ }^{127}$

\section{CNS symptoms}

CNS symptoms are rarely found in ATTR. However, they dominate in a few mutations and often resemble the symptoms of a minor stroke or transient ischemic attack. ${ }^{5,35}$ Dementia, seizures, subarachnoid hemorrhage, ataxia, myelopathy, and deafness may also present as symptoms. Vitreous opacities are often an accompanying complication and are often referred to as the oculoleptomeningeal form of ATTR. On cerebral magnetic resonance examination, the amyloid deposits can be found in the meningeal space. ${ }^{3,36}$

The effect of liver transplantation has generally been disappointing for mutations with CNS engagement and so the procedure is not recommended. No patients with CNS manifestations have been included in clinical trials.

\section{Treatments for ATTR currently approved or on the horizon TTR stabilizers}

The suggestion that TTR amyloid was formed from misfolded monomers raised the question whether stabilization of the TTR tetramer could prevent amyloid formation, and in vitro studies showed that this appeared to be the case. ${ }^{1}$ Nonsteroidal anti-inflammatory drugs appeared to be effective, but only one of the tested compounds, diflunisal, had a strong affinity for TTR - diflunisal is currently being evaluated in a controlled clinical trial. ${ }^{1}$ Tafamidis, a new compound with strong binding affinity and stabilizing properties for TTR, has recently been approved in the European Union for the treatment of ATTR with neuropathy at an early stage of the disease (stage I: patient can walk without support by a stick or crutch). Tafamidis showed disease-modifying properties in an 18-month double-blind clinical trial, followed by an open-label 12-month extension study, through a diminished progression rate of neuropathy in the lower limb (as measured by the Neuropathy Impairment Score in the Lower Limbs), lower deterioration in quality of life for the patient (as measured by the Norfolk Questionnaire), and significantly improved nutritional status of the patient (as measured by the mBMI). ${ }^{29}$ The patients included in the trial were predominantly early-onset cases, a group of patients where full-length TTR amyloid fibrils are most commonly found; therefore, it is not yet known if the response is different for late-onset patients or for patients with other mutations, as has been the case for liver transplantation.

\section{Treatment targeting TTR synthesis}

Since TTR amyloid can be formed from wild-type TTR, as shown to be the case after liver transplantation, treatments aimed to decrease the amount of amyloidogenic protein (ie, variant and wild-type TTR) appear attractive. From transgenic mice experiments, TTR in the cerebral spinal fluid appears to be necessary for normal brain function, ${ }^{128}$ but outside the CNS, TTR is not required to sustain a normal function of vitamin A or thyroid hormone. ${ }^{129}$

To effectively decrease TTR synthesis, two different approaches have been taken: (1) silencing RNA, which interferes with TTR synthesis in the ribosome, and (2) antisense nucleotide, which prevents transcription of messenger RNA 
in the nucleus. Both methods have been utilized in phase I trials and have shown the ability to significantly decrease TTR synthesis. ${ }^{130,131}$

\section{Treatment aimed at removing amyloid deposits}

Serum amyloid $\mathrm{P}$ component (SAP) is found in all amyloid deposits and it appears to protect amyloid from degradation. Recently, an antibody to SAP was shown to be able to degrade amyloid deposits in an amyloid mouse model, and a compound that effectively depressed SAP synthesis in patients with fibrinogen amyloidosis has been developed - CPHPC (R-1-[6-[R-2-carboxy-pyrrolidin-1-yl]-6-oxo-hexanoyl] pyrrolidine-2-carboxylic acid). ${ }^{132,133}$ Treatment with a combination of these two compounds may be effective for all types of amyloid diseases.

Doxycycline is another compound with the ability to dissolve amyloid deposits. ${ }^{134}$ The European Committee for Orphan Medicinal Products has recently designated doxycycline for the treatment of hereditary amyloid polyneuropathy (http://www.emea.europa.eu/docs/en_GB/document_library/ Orphan_designation/2012/05/WC500127736.pdf). The ability of doxycycline to treat ATTR is currently being evaluated in a clinical trial (ClinicalTrials.gov identifier NCT01171859), where doxycycline is combined with tauroursodeoxycholic acid. This combination has proven to be effective in a transgenic mouse model of V30M amyloidosis. ${ }^{135}$

\section{Conclusion}

New insights into the amyloid formation process and the lessons learned from liver transplantation give the opportunity to design potentially effective treatment modalities for ATTR.

Early treatment is required - before the patient suffers from severe, irreversible organ damage - and increased awareness of the disease is an important means of enabling this. Improved and easily accessible diagnostic tools provide the possibility of diagnosing the disease soon after onset by less invasive methods. Noninvasive, readily available tests such as thermal threshold assessment and QST are well suited for peripheral neuropathy. Autonomic neuropathy can be assessed by analysis of beat-to-beat fluctuations in $\mathrm{R}-\mathrm{R}$ intervals. However, for ATTR patients in whom atrial arrhythmias are common, the risk of misinterpretation of the outcome should be taken into consideration, especially in poorly validated deep breathing tests. This is of special importance, since the test is often incorporated into scoring of nerve function in clinical trials.
Heart complications of ATTR have achieved increased recognition as a major problem. DPD scintigraphy together with new echocardiographic methods such as global strain measurements are generally easily available and should be employed for diagnosis and assessment of heart function.

The TTR-stabilizing agent tafamidis appeared to be unable to halt the progress of the disease, even though it markedly diminished the rate of disease progression. So far the approval of tafamidis is restricted to patients with polyneuropathy in an early stage of the disease. For V30M patients with an early onset of the disease, liver transplantation is still a valid treatment option and should be offered to the patients.

The finding of a continuous exchange of amyloid, where variant TTR is rapidly replaced by wild-type TTR in the amyloid deposits after liver transplantation, is encouraging, since it demonstrates that amyloid deposits constantly undergo formation and degradation. If the concentration of the amyloidogenic protein is diminished, the amyloid deposits may subside; this should provide hope for the improvement of patients, especially for those suffering from cardiomyopathy.

It appears reasonable to suspect that a combination of different treatment modalities may be required for the treatment of the disease, and that different treatment regimes will be designed according to the phenotype of the disease.

For ATTR patients and their relatives there is now a solid foundation for optimism, with prospects of several effective medical treatment possibilities within the coming decade.

\section{Acknowledgments}

The authors wish to acknowledge the support received from the following: the Swedish Heart and Lung Foundation (OBS and PL), Central and Clinical ALF grants (OBS and RH), Spearhead grant from Västerbotten County (OBS), the Research Foundation of the Department of Pharmacology and Clinical Neuroscience, Umeå University (EN and VH), and FAMY, FAMY Norrbotten, and the Amyl Foundation (OBS, RH, UW, and EN). The award of a visiting professorship at the Rehabilitation Research Chair, King Saud University, Riyadh, Saudi Arabia, to author EN is gratefully acknowledged.

\section{Disclosure}

OBS has served as a consultant for Pfizer, Alnylam Pharmaceuticals, and Isis Pharmaceuticals companies. OBS has participated as an investigator in clinical trials with tafamidis, diflunisal, and small interfering RNA. 
EN received lecture honoraria from Ipsen and Pfizer. The authors have no other conflicts of interest to report.

\section{References}

1. Hammarström P, Wiseman RL, Powers ET, Kelly JW. Prevention of transthyretin amyloid disease by changing protein misfolding energetics. Science. 2003;299(5607):713-716.

2. Kelly JW, Colon W, Lai Z, et al. Transthyretin quaternary and tertiary structural changes facilitate misassembly into amyloid. Adv Protein Chem. 1997;50:161-181.

3. Benson MD, Kincaid JC. The molecular biology and clinical features of amyloid neuropathy. Muscle Nerve. 2007;36(4):411-423.

4. Connors LH, Lim A, Prokaeva T, Roskens VA, Costello CE. Tabulation of human transthyretin (TTR) variants, 2003. Amyloid. 2003;10(3):160-184.

5. Jacobson DR, Pastore RD, Yaghoubian R, et al. Variant-sequence transthyretin (isoleucine 122) in late-onset cardiac amyloidosis in black Americans. N Engl J Med. 1997;336(7):466-473.

6. Westermark P, Sletten K, Johansson B, Cornwell GG 3rd. Fibril in senile systemic amyloidosis is derived from normal transthyretin. Proc Natl Acad Sci U S A. 1990;87(7):2843-2845.

7. Ueda M, Ageyama N, Nakamura S, et al. Aged vervet monkeys developing transthyretin amyloidosis with the human disease-causing Ile122 allele: a valid pathological model of the human disease. Lab Invest. 2012;92(3):474-484.

8. Coelho T, Sousa A, Lourenço E, Ramalheira J. A study of 159 Portuguese patients with familial amyloidotic polyneuropathy (FAP) whose parents were both unaffected. J Med Genet. 1994;31(4): 293-299.

9. Koike H, Tanaka F, Hashimoto R, et al. Natural history of transthyretin Val30Met familial amyloid polyneuropathy: analysis of lateonset cases from non-endemic areas. J Neurol Neurosurg Psychiatry. 2012;83(2):152-158.

10. Planté-Bordeneuve V, Lalu T, Misrahi M, et al. Genotypicphenotypic variations in a series of 65 patients with familial amyloid polyneuropathy. Neurology. 1998;51(3):708-714.

11. Hellman U, Alarcon F, Lundgren HE, Suhr OB, Bonaiti-Pellié C, Planté-Bordeneuve V. Heterogeneity of penetrance in familial amyloid polyneuropathy, ATTR Val30Met, in the Swedish population. Amyloid. 2008;15(3):181-186.

12. Hörnsten R, Pennlert J, Wiklund U, Lindqvist P, Jensen SM, Suhr OB. Heart complications in familial transthyretin amyloidosis: impact of age and gender. Amyloid. 2010;17(2):63-68.

13. Araki S, Kurihara T, Tawara S, Kuribayashi T. Familial amyloidotic polyneuropathy in Japanese. In: Glenner GG, Pinho e Costa P, de Freitas AF, editors. Amyloid and Amyloidosis. Amsterdam: Excerpta Medica; 1980: $67-77$.

14. Coutinho P, da Silva AM, Lima JK, Barbosa AR. Forty years of experience with type I amyloid neuropathy: review of 483 cases. In: Glenner GG, Pinho e Costa P, de Freitas AF, editors. Amyloid and Amyloidosis. Amsterdam: Excerpta Medica; 1979:88-98.

15. Suhr O, Danielsson A, Holmgren G, Steen L. Malnutrition and gastrointestinal dysfunction as prognostic factors for survival in familial amyloidotic polyneuropathy. J Intern Med. 1994;235(5):479-485.

16. Tashima K, Suhr OB, Ando Y, et al. Gastrointestinal dysfunction in familial amyloidotic polyneuropathy (ATTR Val30Met): comparison of Swedish and Japanese patients. Amyloid. 1999;6(2):124-129.

17. Adams D, Samuel D, Goulon-Goeau C, et al. The course and prognostic factors of familial amyloid polyneuropathy after liver transplantation. Brain. 2000;123(Pt 7):1495-1504.

18. De Carvalho M, Conceição I, Bentes C, Luís ML. Long-term quantitative evaluation of liver transplantation in familial amyloid polyneuropathy (Portuguese V30M). Amyloid. 2002;9(2):126-133.

19. Dubrey SW, Davidoff R, Skinner M, Bergethon P, Lewis D, Falk RH. Progression of ventricular wall thickening after liver transplantation for familial amyloidosis. Transplantation. 1997;64(1):74-80.
20. García-Herola A, Prieto M, Pascual S, et al. Progression of cardiomyopathy and neuropathy after liver transplantation in a patient with familial amyloidotic polyneuropathy caused by tyrosine- 77 transthyretin variant. Liver Transpl Surg. 1999;5(3):246-248.

21. Gustafsson S, Ihse E, Henein MY, Westermark P, Lindqvist P, Suhr OB. Amyloid fibril composition as a predictor of development of cardiomyopathy after liver transplantation for hereditary transthyretin amyloidosis. Transplantation. 2012;93(10):1017-1023.

22. Hörnsten R, Wiklund U, Olofsson BO, Jensen SM, Suhr OB. Liver transplantation does not prevent the development of life-threatening arrhythmia in familial amyloidotic polyneuropathy, Portuguese-type (ATTR Val30Met) patients. Transplantation. 2004;78(1):112-116.

23. Olofsson BO, Backman C, Karp K, Suhr OB. Progression of cardiomyopathy after liver transplantation in patients with familial amyloidotic polyneuropathy, Portuguese type. Transplantation. 2002;73(5):745-751.

24. Pomfret EA, Lewis WD, Jenkins RL, et al. Effect of orthotopic liver transplantation on the progression of familial amyloidotic polyneuropathy. Transplantation. 1998;65(7):918-925.

25. Stangou AJ, Hawkins PN, Heaton ND, et al. Progressive cardiac amyloidosis following liver transplantation for familial amyloid polyneuropathy: implications for amyloid fibrillogenesis. Transplantation. 1998;66(2):229-233.

26. Suhr OB, Herlenius G, Friman S, Ericzon BG. Liver transplantation for hereditary transthyretin amyloidosis. Liver Transpl. 2000;6(3):263-276.

27. Grazi GL, Cescon M, Salvi F, et al. Combined heart and liver transplantation for familial amyloidotic neuropathy: considerations from the hepatic point of view. Liver Transpl. 2003;9(9):986-992.

28. Pilato E, Dell'Amore A, Botta L, Arpesella G. Combined heart and liver transplantation for familial amyloidotic neuropathy. Eur J Cardiothorac Surg. 2007;32(1):180-182.

29. Coelho T, Maia LF, Martins da Silva A, et al. Tafamidis for transthyretin familial amyloid polyneuropathy: a randomized, controlled trial. Neurology. In press 2012.

30. Dyck PJ, Melton LJ 3rd, O’Brien PC, Service FJ. Approaches to improve epidemiological studies of diabetic neuropathy: insights from the Rochester Diabetic Neuropathy Study. Diabetes. 1997;46 Suppl 2: S5-S8.

31. Bergström J, Gustavsson A, Hellman U, et al. Amyloid deposits in transthyretin-derived amyloidosis: cleaved transthyretin is associated with distinct amyloid morphology. J Pathol. 2005;206(2):224-232.

32. Koike H, Ando Y, Ueda M, et al. Distinct characteristics of amyloid deposits in early- and late-onset transthyretin Val30Met familial amyloid polyneuropathy. J Neurol Sci. 2009;287(1-2):178-184.

33. Ihse E, Ybo A, Suhr O, Lindqvist P, Backman C, Westermark P. Amyloid fibril composition is related to the phenotype of hereditary transthyretin V30M amyloidosis. J Pathol. 2008;216(2):253-261.

34. Ihse E, Suhr OB, Hellman U, Westermark P. Variation in amount of wild-type transthyretin in different fibril and tissue types in ATTR amyloidosis. J Mol Med (Berl). 2011;89(2):171-180.

35. Ihse E, Stangou AJ, Heaton ND, et al. Proportion between wild-type and mutant protein in truncated compared to full-length ATTR: an analysis on transplanted transthyretin T60A amyloidosis patients. Biochem Biophys Res Commun. 2009;379(4):846-850.

36. Blevins G, Macaulay R, Harder S, et al. Oculoleptomeningeal amyloidosis in a large kindred with a new transthyretin variant Tyr69His. Neurology. 2003;60(10):1625-1630.

37. Petersen RB, Goren H, Cohen M, et al. Transthyretin amyloidosis: a new mutation associated with dementia. Ann Neurol. 1997;41(3):307-313.

38. Schweitzer K, Ehmann D, Garcia R, Alport E. Oculoleptomeningeal amyloidosis in 3 individuals with the transthyretin variant Tyr69His. Can J Ophthalmol. 2009;44(3):317-319.

39. Uemichi T, Uitti RJ, Koeppen AH, Donat JR, Benson MD. Oculoleptomeningeal amyloidosis associated with a new transthyretin variant Ser64. Arch Neurol. 1999;56(9):1152-1155. 
40. Ikeda S, Nakazato M, Ando Y, Sobue G. Familial transthyretin-type amyloid polyneuropathy in Japan: clinical and genetic heterogeneity. Neurology. 2002;58(7):1001-1007.

41. Sousa A, Andersson R, Drugge U, Holmgren G, Sandgren O. Familial amyloidotic polyneuropathy in Sweden: geographical distribution, age of onset, and prevalence. Hum Hered. 1993;43(5):288-294.

42. Zaros C, Genin E, Hellman U, et al. On the origin of the transthyretin Val30Met familial amyloid polyneuropathy. Ann Hum Genet. 2008;72(Pt 4):478-484.

43. Koike H, Misu K, Ikeda S, et al. Type I (transthyretin Met30) familial amyloid polyneuropathy in Japan: early- vs late-onset form. Arch Neurol. 2002;59(11):1771-1776.

44. Koike H, Misu K, Sugiura M, et al. Pathology of early- vs lateonset TTR Met30 familial amyloid polyneuropathy. Neurology. 2004;63(1):129-138.

45. Olsson M, Hellman U, Planté-Bordeneuve V, Jonasson J, Lång K, Suhr OB. Mitochondrial haplogroup is associated with the phenotype of familial amyloidosis with polyneuropathy in Swedish and French patients. Clin Genet. 2009;75(2):163-168.

46. Bonaïti B, Olsson M, Hellman U, Suhr O, Bonaïti-Pellié C, Planté-Bordeneuve V. TTR familial amyloid polyneuropathy: does a mitochondrial polymorphism entirely explain the parent-oforigin difference in penetrance? Eur J Hum Genet. 2010;18(8): 948-952.

47. Gibbons CH, Illigens BM, Centi J, Freeman R. QDIRT: quantitative direct and indirect test of sudomotor function. Neurology. 2008; 70(24):2299-2304.

48. Novak P. Quantitative autonomic testing. J Vis Exp. 2011;53:2502.

49. Yang NC, Lee MJ, Chao CC, et al. Clinical presentations and skin denervation in amyloid neuropathy due to transthyretin Ala97Ser. Neurology. 2010;75(6):532-538.

50. Ando Y, Yamashita T, Tanaka Y, et al. Role of nitric oxide in the peripheral vessels of patients with familial amyloidotic polyneuropathy (FAP) type I. J Auton Nerv Syst. 1994;50(1):79-85.

51. Dyck PJ, Zimmerman I, Gillen DA, Johnson D, Karnes JL, O’Brien PC. Cool, warm, and heat-pain detection thresholds: testing methods and inferences about anatomic distribution of receptors. Neurology. 1993;43(8):1500-1508.

52. Fruhstorfer H, Lindblom U, Schmidt WC. Method for quantitative estimation of thermal thresholds in patients. J Neurol Neurosurg Psychiatry. 1976;39(11):1071-1075.

53. Gruener G, Dyck PJ. Quantitative sensory testing: methodology, applications, and future directions. J Clin Neurophysiol. 1994;11(6): 568-583.

54. Jamal GA, Hansen S, Weir AI, Ballantyne JP. An improved automated method for the measurement of thermal thresholds: 1 . Normal subjects. J Neurol Neurosurg Psychiatry. 1985;48(4):354-360.

55. Lin YH, Hsieh SC, Chao CC, Chang YC, Hsieh ST. Influence of aging on thermal and vibratory thresholds of quantitative sensory testing. J Peripher Nerv Syst. 2005;10(3):269-281.

56. Rolke R, Baron R, Maier C, et al. Quantitative sensory testing in the German Research Network on Neuropathic Pain (DFNS): standardized protocol and reference values. Pain. 2006;123(3):231-243.

57. Selim MM, Wendelschafer-Crabb G, Hodges JS, et al. Variation in quantitative sensory testing and epidermal nerve fiber density in repeated measurements. Pain. 2010;151(3):575-581.

58. Yarnitsky D. Quantitative sensory testing. Muscle Nerve. 1997;20(2): 198-204.

59. Yarnitsky D, Sprecher E. Thermal testing: normative data and repeatability for various test algorithms. J Neurol Sci. 1994;125(1): 39-45.

60. Kandel ER, Schwartz JH, Jessell TM. Principles of Neural Science. 4th ed. New York: McGraw-Hill/Appleton and Lange; 2000.

61. Schmidt RF, Altner H. Fundamentals of Sensory Physiology. New York (NY): Springer-Verlag; 1978.

62. Becser N, Sand T, Zwart JA. Reliability of cephalic thermal thresholds in healthy subjects. Cephalalgia. 1998;18(8):574-582.
63. Claus D, Hilz MJ, Hummer I, Neundörfer B. Methods of measurement of thermal thresholds. Acta Neurol Scand. 1987;76(4): 288-296.

64. Heldestad V, Linder J, Sellersjö L, Nordh E. Reproducibility and influence of test modality order on thermal perception and thermal pain thresholds in quantitative sensory testing. Clin Neurophysiol. 2010;121(11): 1878-1885

65. Hilz MJ, Glorius S, Berić A. Thermal perception thresholds: influence of determination paradigm and reference temperature. J Neurol Sci. 1995;129(2):135-140.

66. Levy D, Abraham R, Reid G. A comparison of two methods for measuring thermal thresholds in diabetic neuropathy. J Neurol Neurosurg Psychiatry. 1989;52(9):1072-1077.

67. Lowenstein L, Jesse K, Kenton K. Comparison of perception threshold testing and thermal-vibratory testing. Muscle Nerve. 2008;37(4): 514-517.

68. Meier PM, Berde CB, DiCanzio J, Zurakowski D, Sethna NF. Quantitative assessment of cutaneous thermal and vibration sensation and thermal pain detection thresholds in healthy children and adolescents. Muscle Nerve. 2001;24(10): 1339-1345.

69. Verdugo R, Ochoa JL. Quantitative somatosensory thermotest: a key method for functional evaluation of small calibre afferent channels. Brain. 1992;115(Pt 3):893-913

70. Heldestad V, Nordh E. Quantified sensory abnormalities in early genetically verified transthyretin amyloid polyneuropathy. Muscle Nerve. 2007;35(2):189-195.

71. Hsu W, Bettegowda C, Jallo GI. Intramedullary spinal cord tumor surgery: can we do it without intraoperative neurophysiological monitoring? Childs Nerv Syst. 2010;26(2):241-245.

72. Kortelainen P, Puranen J, Koivisto E, Lähde S. Symptoms and signs of sciatica and their relation to the localization of the lumbar disc herniation. Spine (Phila Pa 1976). 1985;10(1):88-92.

73. Travell JG, Simons DG. Myofascial Pain and Dysfunction. Philadelphia (PA): Lippincott Williams \& Wilkins; 1992

74. Vallbo AB, Hagbarth KE, Wallin BG. Microneurography: how the technique developed and its role in the investigation of the sympathetic nervous system. J Appl Physiol. 2004;96(4):1262-1269.

75. Esler M. Clinical application of noradrenaline spillover methodology: delineation of regional human sympathetic nervous responses. Pharmacol Toxicol. 1993;73(5):243-253.

76. Low PA, editor. Clinical Autonomic Disorders: Evaluation and Management. Boston (MA): Little Brown and Co; 1993.

77. Low PA, Caskey PE, Tuck RR, Fealey RD, Dyck PJ. Quantitative sudomotor axon reflex test in normal and neuropathic subjects. Ann Neurol. 1983;14(5):573-580.

78. Wang AK, Fealey RD, Gehrking TL, Low PA. Patterns of neuropathy and autonomic failure in patients with amyloidosis. Mayo Clin Proc. 2008;83(11):1226-1230.

79. Delahaye N, Rouzet F, Sarda L, et al. Impact of liver transplantation on cardiac autonomic denervation in familial amyloid polyneuropathy. Medicine (Baltimore). 2006;85(4):229-238.

80. Hörnsten R, Suhr OB, Jensen SM, Wiklund U. Outcome of heart rate variability and ventricular late potentials after liver transplantation for familial amyloidotic polyneuropathy. Amyloid. 2008;15(3): 187-195.

81. Kinoshita O, Hongo M, Saikawa Y, et al. Heart rate variability in patients with familial amyloid polyneuropathy. Pacing Clin Electrophysiol. 1997;20(12 Pt 1):2949-2953.

82. Wiklund U, Hörnsten R, Karlsson M, Suhr OB, Jensen SM. Abnormal heart rate variability and subtle atrial arrhythmia in patients with familial amyloidotic polyneuropathy. Ann Noninvasive Electrocardiol. 2008;13(3):249-256.

83. Wiklund U, Hörnsten R, Olofsson BO, Suhr OB. Cardiac autonomic function does not improve after liver transplantation for familial amyloidotic polyneuropathy. Auton Neurosci. 2010;156(1-2): 124-130. 
84. Abe M, Ando Y, Higashi K, Kano T. Non-neurogenic periodic fluctuations in heart rate and vasomotion appearing in familial amyloid polyneuropathy (FAP) type I (Met30). J Auton Nerv Syst. 1996;60(1-2):71-75.

85. Hörnsten R, Suhr OB, Olofsson BO, Wiklund U. Arrhythmia: a pitfall in tests of cardiac autonomic function after liver transplantation for familial amyloidotic polyneuropathy; a long-term follow-up of Swedish patients. Amyloid. 2012;19(2):81-86.

86. Obayashi K, Hörnsten R, Wiklund U, et al. Blood pressure overshoot after tilt reversal in patients with familial amyloidotic polyneuropathy. Hypertens Res. 2011;34(1):133-138.

87. Zhao Y, Hörnsten R, Lindqvist P, Wiklund U, Suhr OB, Henein MY. Left ventricular dyssynchrony is associated with reduced heart rate variability in familial amyloidotic polyneuropathy. Int J Cardiol 2012;155(2):273-278.

88. Suhr OB, Lindqvist P, Olofsson BO, Waldenström A, Backman C. Myocardial hypertrophy and function are related to age at onset in familial amyloidotic polyneuropathy. Amyloid. 2006;13(3):154-159.

89. Rapezzi C, Riva L, Quarta CC, et al. Gender-related risk of myocardial involvement in systemic amyloidosis. Amyloid. 2008;15(1):40-48.

90. Bhandari AK, Nanda NC. Myocardial texture characterization by two-dimensional echocardiography. Am J Cardiol. 1983;51(5): $817-825$.

91. Falk RH, Plehn JF, Deering T, et al. Sensitivity and specificity of the echocardiographic features of cardiac amyloidosis. Am J Cardiol. 1987;59(5):418-422.

92. St John Sutton MG, Reichek N, Kastor JA, Giuliani ER. Computerized M-mode echocardiographic analysis of left ventricular dysfunction in cardiac amyloid. Circulation. 1982;66(4):790-799.

93. Chandrasekaran K, Aylward PE, Fleagle SR, et al. Feasibility of identifying amyloid and hypertrophic cardiomyopathy with the use of computerized quantitative texture analysis of clinical echocardiographic data. J Am Coll Cardiol. 1989;13(4):832-840.

94. Eriksson P, Backman C, Eriksson A, Eriksson S, Karp K, Olofsson BO. Differentiation of cardiac amyloidosis and hypertrophic cardiomyopathy: a comparison of familial amyloidosis with polyneuropathy and hypertrophic cardiomyopathy by electrocardiography and echocardiography. Acta Med Scand. 1987;221(1):39-46.

95. Lindqvist P, Olofsson BO, Backman C, Suhr O, Waldenström A. Pulsed tissue Doppler and strain imaging discloses early signs of infiltrative cardiac disease: a study on patients with familial amyloidotic polyneuropathy. Eur J Echocardiogr. 2006;7(1):22-30.

96. Engvall C, Henein M, Holmgren A, Suhr OB, Mörner S, Lindqvist P. Can myocardial strain differentiate hypertrophic from infiltrative etiology of a thickened septum? Echocardiography. 2011;28(4): $408-415$.

97. Migrino RQ, Harmann L, Woods T, Bright M, Truran S, Hari P. Intraventricular dyssynchrony in light chain amyloidosis: a new mechanism of systolic dysfunction assessed by 3-dimensional echocardiography. Cardiovasc Ultrasound. 2008;6:40.

98. Rapezzi C, Quarta CC, Guidalotti PL, et al. Usefulness and limitations of 99mTc-3,3-diphosphono-1,2-propanodicarboxylic acid scintigraphy in the aetiological diagnosis of amyloidotic cardiomyopathy. Eur $J$ Nucl Med Mol Imaging. 2011;38(3):470-478.

99. Falk RH, Lee VW, Rubinow A, Skinner M, Cohen AS. Cardiac technetium-99m pyrophosphate scintigraphy in familial amyloidosis. Am J Cardiol. 1984;54(8):1150-1151.

100. Hongo M, Hirayama J, Fujii T, et al. Early identification of amyloid heart disease by technetium-99m- pyrophosphate scintigraphy: a study with familial amyloid polyneuropathy. Am Heart J. 1987;113(3): 654-662.

101. Perugini E, Rapezzi C, Piva T, et al. Non-invasive evaluation of the myocardial substrate of cardiac amyloidosis by gadolinium cardiac magnetic resonance. Heart. 2006;92(3):343-349.

102. Palladini G, Campana C, Klersy C, et al. Serum N-terminal pro-brain natriuretic peptide is a sensitive marker of myocardial dysfunction in AL amyloidosis. Circulation. 2003;107(19):2440-2445.
103. Suhr OB, Anan I, Backman C, et al. Do troponin and B-natriuretic peptide detect cardiomyopathy in transthyretin amyloidosis? J Intern Med. 2008;263(3):294-301.

104. Palladini G, Barassi A, Klersy C, et al. The combination of highsensitivity cardiac troponin $\mathrm{T}$ (hs-cTnT) at presentation and changes in N-terminal natriuretic peptide type B (NT-proBNP) after chemotherapy best predicts survival in AL amyloidosis. Blood. 2010;116(18):3426-3430.

105. Eriksson A, Eriksson P, Olofsson BO, Thornell LE. The cardiac atrioventricular conduction system in familial amyloidosis with polyneuropathy: a clinico-pathologic study of six cases from northern Sweden. Acta Pathol Microbiol Immunol Scand A. 1983;91(5):343-349.

106. Eriksson A, Eriksson P, Olofsson BO, Thornell LE. The sinoatrial node in familial amyloidosis with polyneuropathy: a clinico-pathological study of nine cases from northern Sweden. Virchows Arch A Pathol Anat Histopathol. 1984;402(3):239-246.

107. Ando Y, Suhr OB. Autonomic dysfunction in familial amyloidotic polyneuropathy (FAP). Amyloid. 1998;5(4):288-300.

108. Niklasson U, Olofsson BO, Bjerle P. Autonomic neuropathy in familial amyloidotic polyneuropathy: a clinical study based on heart rate variability. Acta Neurol Scand. 1989;79(3):182-187.

109. Beckman A, Bjerle P, Olofsson B. Electrocardiographic findings in familial amyloidotic polyneuropathy. Am J Noninvas Cardiol. 1992;6:192-196.

110. De Freitas AF. The heart in Portuguese amyloidosis. Postgrad Med J. 1986;62(728):601-605.

111. De Freitas AF, Barbedo A. Conduction disturbances in 190 patients with familial amyloidotic polyneuropathy (Andrade's type). Adv Cardiol. 1978;21:206-209.

112. Sawayama T, Kurihara T, Araki S. Noninvasive cardiovascular findings in familial amyloid polyneuropathy. Br Heart J. 1978;40(11): 1288-1292.

113. Okamoto S, Hörnsten R, Obayashi K, Wijayatunga P, Suhr OB. Continuous development of arrhythmia is observed in Swedish transplant patients with familial amyloidotic polyneuropathy (amyloidogenic transthyretin Val30Met variant). Liver Transpl. 2011;17(2):122-128.

114. Hörnsten R, Wiklund U, Suhr OB, Jensen SM. Ventricular late potentials in familial amyloidotic polyneuropathy. J Electrocardiol. 2006;39(1):57-62.

115. Anan I, El-Salhy M, Ando Y, et al. Colonic enteric nervous system in patients with familial amyloidotic neuropathy. Acta Neuropathol. 1999;98(1):48-54.

116. Anan I, El-salhy M, Ando Y, Terazaki H, Suhr OB. Comparison of amyloid deposits and infiltration of enteric nervous system in the upper with those in the lower gastrointestinal tract in patients with familial amyloidotic polyneuropathy. Acta Neuropathol. 2001;102(3):227-232.

117. Suhr OB, Ericzon BG, Friman S. Long-term follow-up of survival of liver transplant recipients with familial amyloid polyneuropathy (Portuguese type). Liver Transpl. 2002;8(9):787-794.

118. Berk JL, Dyck PJ, Obici L, et al. The diflunisal trial: update on study drug tolerance and disease progression. Amyloid. 2011;18 Suppl 1: 191-192

119. Lång K, Wikström L, Danielsson A, Tashima K, Suhr OB. Outcome of gastrointestinal complications after liver transplantation for familial amyloidotic polyneuropathy. Scand J Gastroenterol. 2000;35(9):985-989.

120. Lobato L, Beirão I, Silva M, et al. End-stage renal disease and dialysis in hereditary amyloidosis TTR V30M: presentation, survival and prognostic factors. Amyloid. 2004;11(1):27-37.

121. Lobato L, Ventura A, Beirão I, et al. End-stage renal disease in familial amyloidosis ATTR Val30Met: a definitive indication to combined liver-kidney transplantation. Transplant Proc. 2003;35(3):1116-1120.

122. Nowak G, Suhr OB, Wikström L, Wilczek H, Ericzon BG. The longterm impact of liver transplantation on kidney function in familial amyloidotic polyneuropathy patients. Transpl Int. 2005;18(1):111-115. 
123. Ando E, Ando Y, Okamura R, Uchino M, Ando M, Negi A. Ocular manifestations of familial amyloidotic polyneuropathy type I: longterm follow up. Br J Ophthalmol. 1997;81(4):295-298.

124. Ando E, Ando Y, Haraoka K. Ocular amyloid involvement after liver transplantation for polyneuropathy. Ann Intern Med. 2001;135(10): 931-932.

125. Sandgren O, Kjellgren D, Suhr OB. Ocular manifestations in liver transplant recipients with familial amyloid polyneuropathy. Acta Ophthalmol. 2008;86(5):520-524.

126. Svendsen IH, Steensgaard-Hansen F, Nordvåg BY. A clinical, echocardiographic and genetic characterization of a Danish kindred with familial amyloid transthyretin methionine 111 linked cardiomyopathy. Eur Heart J. 1998;19(5):782-789.

127. Suhr OB, Andersen O, Aronsson T, et al. Report of five rare or previously unknown amyloidogenic transthyretin mutations disclosed in Sweden. Amyloid. 2009;16(4):208-214.

128. Buxbaum JN, Ye Z, Reixach N, et al. Transthyretin protects Alzheimer's mice from the behavioral and biochemical effects of Abeta toxicity. Proc Natl Acad Sci U S A. 2008;105(7):2681-2686.

129. Episkopou V, Maeda S, Nishiguchi S, et al. Disruption of the transthyretin gene results in mice with depressed levels of plasma retinol and thyroid hormone. Proc Natl Acad Sci U S A. 1993;90(6):2375-2379.
130. Bodin K, Ellmerich S, Kahan MC, et al. Antibodies to human serum amyloid P component eliminate visceral amyloid deposits. Nature. 2010;468(7320):93-97.

131. Coelho T, Suhr OB, Adams D et al. Phase I safety, pharmacokinetic and pharmacodynamics results for ALN-TTR01, a novel RNAi therapeutic for the treatment of transthyretin amyloidosis (abstract). VIIIth International Symposium on Familial Amyloidotic Polyneuropathy, Kumamoto, Japan 2011, P62

132. Monia B, Ackerman E, Guo S, Benson MD. Clinical development of an antisense therapy for the treatment of transthyretin-associated polyneuropathy (abstract). VIIIth International Symposium on Familial Amyloidotic Polyneuropathy, Kumamoto, Japan 2011, P64

133. Gillmore JD, Tennent GA, Hutchinson WL, et al. Sustained pharmacological depletion of serum amyloid $\mathrm{P}$ component in patients with systemic amyloidosis. Br J Haematol. 2010;148(5):760-767.

134. Cardoso I, Merlini G, Saraiva MJ. 4'-Iodo-4'-deoxydoxorubicin and tetracyclines disrupt transthyretin amyloid fibrils in vitro producing noncytotoxic species: screening for TTR fibril disrupters. FASEB J. 2003;17(8):803-809.

135. Cardoso I, Martins D, Ribeiro T, Merlini G, Saraiva MJ. Synergy of combined doxycycline/TUDCA treatment in lowering transthyretin deposition and associated biomarkers: studies in FAP mouse models. J Transl Med. 2010;8:74.
Degenerative Neurological and Neuromuscular Disease

\section{Publish your work in this journal}

Degenerative Neurological and Neuromuscular Disease is an international, peer-reviewed, open access journal focusing on research into degenerative neurological and neuromuscular disease, identification of therapeutic targets and the optimal use of preventative and integrated treatment interventions to achieve improved outcomes, enhanced

\section{Dovepress}

survival and quality of life for the patient. The manuscript management system is completely online and includes a very quick and fair peer-review system. Visit http://www.dovepress.com/testimonials.php to read real quotes from published authors. 